\title{
Ethical, legal, and social implications of incorporating genomic information into electronic health records
}

\author{
Ribhi Hazin, MD1, Kyle B. Brothers, MD2,3, Bradley A. Malin, PhD ${ }^{4-6}$, Barbara A. Koenig, PhD ${ }^{7,8}$, \\ Saskia C. Sanderson, PhD ${ }^{9}$, Mark A. Rothstein, JD ${ }^{3}$, Marc S. Williams, MD ${ }^{10}$, Ellen W. Clayton, JD, MD ${ }^{11}$ \\ and Iftikhar J. Kullo, MD ${ }^{12}$
}

The inclusion of genomic data in the electronic health record raises important ethical, legal, and social issues. In this article, we highlight these challenges and discuss potential solutions. We provide a brief background on the current state of electronic health records in the context of genomic medicine, discuss the importance of equitable access to genome-enabled electronic health records, and consider the potential use of electronic health records for improving genomic literacy in patients and providers. We highlight the importance of privacy, access, and security, and of determining which genomic information is included in the electronic health record. Finally, we discuss the challenges of reporting incidental findings, storing and reinterpreting genomic data, and nondocumentation and duty to warn family members at potential genetic risk.

Genet Med advance online publication 12 September 2013

Key Words: clinical decision support, electronic health records; ethical, legal, and social implications; genomics; personalized medicine
Recent advances, including the advent of massively parallel sequencing, have made genomic medicine a realistic goal. ${ }^{1}$ Genomic profiles are expected to enable clinicians to individualize care and thus minimize the variation in outcomes that can result from "one-size-fits-all" approaches. ${ }^{2,3}$ Genome sequencing is already being used for the diagnosis of uncharacterized disorders. ${ }^{4}$ Somatic and tumor genome sequencing, as well as gene expression profiling, are being used to understand cancer behavior and response to treatment. ${ }^{5-11}$ However, to optimally use genomic information for diagnosis, treatment, and prevention of disease, suitably designed electronic health record (EHR) systems will be needed. ${ }^{12,13}$

Although paper records and human interpretation of laboratory results have sufficed in the past, the synthesis and interpretation of large amounts of complex genomic information will require electronic tools capable of delivering to patients and providers the right amount and types of information at point of care. It is only through such tools that we can realize the potential of genomic medicine to improve patient-care outcomes and lower health-care costs. ${ }^{14}$ For example, if genetic testing reveals a patient to be at increased risk of developing early-onset colon cancer, an alert in the EHR could notify the patient's primarycare physician and suggest appropriate interventions, including colonoscopy initiated at an earlier age and performed more frequently. The EHR could also generate patient-focused informational literature to increase awareness of the early symptoms of this condition and potentially increase compliance with surveillance procedures.

The inclusion of genomic data in the EHR, however, raises important ethical, legal, and social issues. In this article, we highlight these challenges and discuss potential solutions. We provide a brief background on the current state of EHRs in the context of genomic medicine, discuss the importance of equitable access to genome-enabled EHRs, and consider the potential use of EHRs for improving genomic literacy in patients and providers. We highlight the importance of privacy, access, and security, and of determining which genomic information is included in the EHR. Finally, we discuss the challenges of reporting incidental findings, storing and reinterpreting genomic data, and nondocumentation and duty to warn family members at potential genetic risk.

\section{EHRS AND GENOMIC MEDICINE: THE CURRENT STATE}

In 2009, only 1.5\% of American hospitals utilized comprehensive EHR systems capable of clinical decision support applications. ${ }^{15}$

\footnotetext{
${ }^{1}$ Department of Internal Medicine, Wayne State University School of Medicine, Detroit, Michigan, USA; ${ }^{2}$ Department of Pediatrics, University of Louisville School of Medicine, Louisville, Kentucky, USA; ${ }^{3}$ Institute for Bioethics, Health Policy, and Law, University of Louisville School of Medicine, Louisville, Kentucky, USA; ${ }^{4}$ Department of Biomedical Informatics, School of Medicine, Vanderbilt University, Nashville, Tennessee, USA; ${ }^{5}$ Department of Electrical Engineering and Computer Science, School of Engineering, Vanderbilt University, Nashville, Tennessee, USA; ${ }^{6}$ Center for Biomedical Ethics and Society, Vanderbilt University, Nashville, Tennessee, USA; ${ }^{7}$ Department of Social and Behavioral Sciences, Institute for Health and Aging, University of California, San Francisco, San Francisco, California, USA; ${ }^{8}$ Department of Anthropology, History, and Social Medicine, University of California, San Francisco, San Francisco, California, USA; ${ }^{9}$ Genetics and Genomic Sciences, The Charles Bronfman Institute for Personalized Medicine, Icahn School of Medicine at Mount Sinai, New York, New York, USA; ${ }^{10}$ Genomic Medicine Institute, Geisinger Health System, Danville, Pennsylvania, USA; ${ }^{11}$ Center for Biomedical Ethics and Society, Vanderbilt University, Nashville, Tennessee, USA; ${ }^{12}$ Division of Cardiovascular Diseases, Mayo Clinic, Rochester, Minnesota, USA. Correspondence: Iftikhar J. Kullo (kullo.iftikhar@mayo.edu) or Ellen W.Clayton (ellen.clayton@vanderbilt.edu)
} 
By 2012, the proportion had increased to $12 \%$, with the majority located in urban areas. ${ }^{16}$ Clinical decision support capability within various EHR systems varies, and this may negatively impact health outcomes and potentially heighten disparities. The Health Information Technology for Economic and Clinical Health Act of 2009 includes several incentives to increase the use of EHR systems by the federally mandated 2013 deadline. Furthermore, federal guidelines mandate compliance with a broad set of "meaningful use" objectives as a prerequisite for certifying EHR systems. Adoption of meaningful use objectives will require EHR systems to deploy greater storage and processing capabilities that may improve the efficiency, quality, and safety of health-care delivery. ${ }^{17}$

Genetic tests are currently conducted as single-gene or candidate-gene panels, and typically, the genotyping report from the laboratory is scanned and placed as a PDF file in the EHR. Such a process will be inadequate as we scale up to using genome/ exome sequencing in the clinical setting. Genome sequencing has demonstrated utility in various clinical settings and will likely become increasingly integrated into clinical practice in the future. ${ }^{18,19}$ Existing EHR systems do not yet include the tools necessary for annotating, mining, and analyzing genomic information over the course of a patient's life span. EHR systems will need to be remodeled to integrate results of genome sequencing with other clinical information, preferably by leveraging existing informatics networks. ${ }^{20}$ In particular, EHR systems will need to include genomic clinical decision support tools to allow clinicians to practice genomic medicine at point of care.

\section{EHRS AND EQUITABLE ACCESS TO GENOMIC MEDICINE}

Health disparities that persist across demographic groups have proven to be a refractory problem in the American health-care system. There is hope that genomic medicine might reduce health disparities by ensuring that health interventions are tailored to an individual's genetic makeup, rather than less precise modifications in care based on race or ethnicity. ${ }^{21}$ These mitigating effects on health disparities will only be possible, however, if patients have comparable access to genomic testing, as well as to EHRs with integrated genomic clinical decision support, and to the subsequent interventions indicated by genomic results. Indeed, Francis Collins, ${ }^{22}$ director of the National Institutes of Health, among others, has suggested that genome sequencing should be available to all patients in the United States. However, this egalitarian vision for access to genome sequencing needs to include also a commitment to providing EHR decision support of uniform quality, as well as the clinical care needed.

Improved access to genome sequencing and informatics pipelines without commensurate access to well-designed decision support for physicians may lead to poor understanding of the results and their implications by physicians, as well as inappropriate actions which could harm rather than benefit the patient. Inadequate focus on incorporating genomic information into EHRs with appropriate clinical decision support and downstream interventions could also compromise public trust more broadly and hinder the use of genome sequencing in clinical practice. The full benefit of EHR-supported genomic medicine may depend on patients accessing and acting on genomic information reported through online patient portals. Lack of Internet access may limit the benefits of genomic medicine from reaching the poor, especially those residing in rural areas. Recognizing other socioeconomic or health system barriers among patients with low literacy can reduce obstacles in integrating genomics into clinical care. In keeping with the principle of justice, policies that strive to ensure affordable, fair, and just allocation of genomic medicine should be encouraged. It would be tragic if genome sequencing led to greater, not fewer, health disparities.

\section{IMPROVING GENOMIC LITERACY AMONG PATIENTS}

To promote patient participation in genomics, the US Department of Health and Human Services launched the Personalized Health Care Initiative Policy to facilitate patient empowerment through genomics as a central priority of future health-care programs. ${ }^{23}$ It is hoped that broad public engagement campaigns will encourage public discourse on genomics as well as improve the ability of patients to make informed decisions regarding their personalized care. For example, online educational videos can be used to engage the public and to help patients better understand how genomics relates to their health. ${ }^{24}$ For optimal utilization of genomic information in the clinical setting, patients should be aware of the benefits and limitations of genomic testing. Active patient participation could also be promoted by interactive tools that allow patients to provide family history and additional information that will facilitate interpretation of genomic testing.

EHRs may also evolve to increasingly acknowledge and respond to patient-driven preferences using patient portals and secure mobile device connections. Through remote monitoring, text messaging, automated alerts, apps, and other forms of digital communication, mobile devices are poised to transform the delivery of health care by enhancing communication between patients and their providers. In the "Open Notes" project, ${ }^{25}$ allowing patients secure access to their clinical notes was beneficial from the patients' perspective. Health-care interactions that emphasize broader patient engagement and improved communication with such technologies will facilitate the integration of genomics into health-care systems.

Sociocultural, economic, personal, and familial factors can influence the decisions of patients related to genomic testing. Significant resources will need to be allocated in order to provide genetic counseling services. Yet there are only 3,026 board-certified genetic counselors in the United States, and little effort is under way to expand genetic counseling programs ${ }^{26}$ The lack of genetic counselors to provide such services may impede the use of genomic testing in clinical 
practice. Modules incorporated into the EHRs or patient portals may substitute for or lessen the burden of genetic counseling. Improved genomic literacy and genomics training among nurses will also be important in the establishment of effective interventions for the provision of genetic counseling and public education. ${ }^{27}$ EHR applications that improve coordination between patients and their health-care providers may increase patients' participation in their own care and contribute toward meaningful use of EHR systems. ${ }^{28}$ Although such tools may empower patients, it is currently unknown whether these tools can, in fact, replace direct advice from health-care providers, especially for patients whose understanding or access to technology may limit their ability to effectively utilize results reported outside the traditional clinical encounter. ${ }^{29}$

\section{IMPROVING GENOMIC LITERACY AMONG CARE PROVIDERS}

Primary-care physicians will likely be on the front line of using genomic medicine in mainstream care, yet numerous studies have demonstrated that many physicians lack the requisite knowledge of genomics needed to provide adequate genomic testing and counseling. ${ }^{30-32} \mathrm{~A}$ recent survey of 10,000 American physicians conducted by the American Medical Association found that only $26 \%$ understood how genetics tests could influence treatment decisions, and only $10 \%$ felt adequately trained to integrate genetic testing into their practice. ${ }^{33}$ The new dimension of genome-informed medical care is likely to further unmask knowledge gaps in this area and increase the frequency of misinterpretations, thereby exposing patients to medical risk. A great deal of work is needed to ensure that primary-care providers are prepared for the era of genomic medicine. Appropriately designed EHR systems, combined with specialized training on interpreting genomic results, ${ }^{34}$ may be able to mitigate some of these concerns. Genomic clinical decision support is enabling providers to practice genomic medicine at point of care. ${ }^{35}$

Even if providers can build competence in working with genomic test results, they could still face difficulty finding time to address all of the relevant findings. ${ }^{36}$ The importance of robust EHR systems for ensuring efficient patient care is highlighted by a recent study that found that without an EHR to mitigate their workload, the average physician would take up to $5 \mathrm{~h}$ to adequately inform a typical patient of his or her genomic risk profile. ${ }^{37}$ EHRs can be equipped with algorithms to interpret genomic tests and facilitate the physician's ability to provide genome-informed clinical care. ${ }^{38,39}$ Standardized methods for reporting genomic tests will similarly facilitate implementation of genomic medicine. A genetic test template has been proposed that would provide a more uniform approach to interpretation. ${ }^{40,41}$ Such a template may facilitate a more standardized approach to genomic testing, enable primary-care providers to better communicate genomic results to patients, and reduce errors associated with the reporting of results to patients. ${ }^{40,41}$

\section{MAINTAINING PRIVACY IN THE SETTING OF INTEROPERABLE EHR SYSTEMS}

Because genomic medicine will depend on the aggregation of vast amounts of genomic information, effective implementation of genomic medicine will require EHR systems that can exchange information in real time via data-sharing functions. ${ }^{42}$ In an effort to ensure interoperability of EHR systems, the Office of Standards and Interoperability at the US Department of Health and Human Services has mandated compliance with validated national standards to enable coordination of care between various stakeholders. ${ }^{43}$ Interoperable EHRs can allow access to patient information across the continuum of care and reduce medical errors and improve the delivery of health care.

As is true for all personal health information, the use of genomic data must be balanced against the need to prevent disclosure of sensitive information. Sharing of genomic information mandates security measures that protect not only the information stored within a single health-care institution but also the exchange of information among institutions and with patients themselves. No point of vulnerability raises more challenges than the connections that allow components of EHR systems within institutions and at different institutions to interoperate. If interoperable EHR systems are to maintain privacy, appropriate access controls and security measures must be implemented throughout the workflow. ${ }^{44}$

Unlike traditional paper medical records, EHR systems can be programmed to include encrypted information, audit functions, ${ }^{45}$ and other safeguards to maintain security of health records. No form of electronic security, however, can prevent individuals who view the exchanged data from improperly revealing sensitive information. For this reason, the security of data exchanged between interoperable EHRs must be enforced through institutional policies and interinstitutional data use agreements. Criminal sanctions, monetary penalties, disciplinary action by licensing boards, or loss of employment, among other consequences, may be necessary for deterring future breaches.

\section{PATIENT ACCESS AND DATA SECURITY}

Although private health information should be protected according to accepted guidelines, once a patient has obtained his or her own health information, the patient shares responsibility for ensuring the confidentiality, privacy, and safety of that information. At a time when patients are increasingly demanding access to their individual genomic results, ${ }^{46}$ it is critical that patient portals that can deliver this information do not also serve as entry points for both high-tech and mundane breaches. For example, unauthorized individuals may gain access to genomic results when patients inadvertently leave browsers logged in to patient portals. Because the loss of a mobile device or other actions by patients could cause personal health information to be revealed, educating patients in the proper use of patient portals will be an important adjunct to higher-tech security measures. 


\section{DETERMINING WHICH GENOMIC INFORMATION TO INCLUDE IN THE EHR}

It is not yet clear how the results of genomic sequencing will be incorporated into the EHR and, in particular, linked to clinical decision support. Should all results of potential clinical significance be reported or just the actionable results? What content should be included in the results? The American College of Medical Genetics and Genomics (ACMG) recently recommended that whenever genome sequencing is conducted, 56 variants related primarily to cancers and cardiac conditions should be reported back from the laboratory to the physician, recognizing that this list will evolve and grow over time. ${ }^{47}$ Of note, the ACMG chose not to make recommendations regarding pharmacogenomic variants, even though efforts to incorporate pharmacogenomic information into the EHR are already under way. ${ }^{48}$ EHR systems are being programmed to recommend specific modifications to prescriptions or other orders based on a patient's genetic predisposition, or even to intercept orders when genetic information indicates that risk for an adverse effect is high. ${ }^{49}$ The Electronic Medical Records and Genomics Network is developing initiatives to harness EHR systems for tailored drug therapy; ${ }^{48}$ network members at Vanderbilt University Medical Center recently launched the Pharmacogenomic Resource for Enhanced Decisions in Care and Treatment program to enable optimal drug dosing and reduce adverse drug reactions. ${ }^{50-52}$ For example, within this program, guidelines are provided to caregivers regarding clopidogrel dosing or alternative therapy in patients with the CYP2C19 ${ }^{\star} 2{ }^{\star} 2$ genotype, which is associated with a reduced response to clopidogrel.

Clinical decision support tools, including "pop-ups," can provide immediate data that can guide clinicians on health-related decisions and also help physicians adhere to evidence-based guidelines and improve health-care outcomes. Although practice guidelines may not be applicable to all patients, in instances in which such guidelines can improve outcomes, such EHR functions may be useful. A major obstacle to creating genomic clinical decision support is lack of standardized nomenclature for genetic variants. Currently, several groups, including the Health Level 7 Genomics Work Group, are attempting to address this challenge. ${ }^{53}$

\section{INCIDENTAL GENOMIC FINDINGS}

Whole-genome sequencing can yield as many as 50,000 novel gene variants per individual. ${ }^{54}$ Although most of these variations are benign, a small proportion may potentially confer significantly higher risk of disease. In a recent study of 141 patients in 16 focus groups, Murphy et al. ${ }^{55}$ found that participants involved in genetic studies overwhelmingly preferred to be informed of their results from genetic tests. Similar results have been generated in studies involving genomic testing in clinical settings. ${ }^{38,56}$ Future guidelines focused on the appropriate reporting of incidental findings (IFs) may encourage health-care providers to harness the analytical capacity of EHR systems for implementing reporting criteria, processing, and disseminating IFs. Many groups have focused on addressing these issues, including the National Human Genome Research Institute-funded Return of Results consortium and the ACMG.

The recommendations of the ACMG that certain IFs should be returned from the laboratory, at least to the physician if not necessarily to the patient, have stimulated considerable debate. ${ }^{47}$ Even if the consensus is that certain highly actionable results should be returned, there will still be a need to acknowledge patient preferences in the majority of cases. ${ }^{57}$ For example, patients should be allowed to decide if they would like to be informed about their risk for developing untreatable conditions such as Alzheimer disease. Notwithstanding patient preferences, as IFs obtained in genomic testing become more frequent, notifying patients may no longer be feasible; ${ }^{58}$ indeed, this is at the crux of the ACMG recommendations. Furthermore, most IFs may lack clinical relevance, and it remains debatable whether patients should be informed of such findings. ${ }^{59-62}$ The ACMG and the National Human Genome Research Institute are currently spearheading efforts to adopt innovative approaches for guiding clinicians on appropriate reporting of IFs in appropriate ways, with varying levels of focus on reflecting patient preferences.

With respect to the design of EHR systems for reporting IFs, there are currently no standardized protocols for synthesizing, analyzing, and disclosing IFs to patients or providers in a manner that is consistent with the aims of routine patient care. Before such protocols can be developed, there is a need for consensus on who is responsible for returning genomic results and whether results should be returned to the patient, the patient's physician, or both. In the absence of effective EHR-based reporting tools, clinicians could be burdened with the daunting task of deciding which IFs should be reported to patients and which should lead to changes in care, a responsibility they may not have the expertise or appropriate training to fulfill. ${ }^{61,63}$ Although the ACMG guidelines were designed in part to circumvent some of these challenges, much burden would still remain on the health-care provider regarding clinical decisions based on IFs and communication of IFs to the patient. EHRlinked expert clinical decision support can potentially mitigate this burden in the future. Some experts caution that haphazardly returning results to patients may change the "standard of care" and thus legally bind providers to return results in the future ${ }^{59}$ These realities highlight the need for controlled studies focused on developing best practices for disclosure of results from genomic studies, as well as for continued debate regarding the ACMG and other emerging guidelines.

In the medium to long term, how to tackle the potentially overwhelming burden of responding to large numbers of genomic results will need to be addressed by a wide array of stakeholders, including medical geneticists, molecular geneticists, bioethicists, and payers. The classification and identification of IFs at the local level will need to consider the work of national and international groups such as the ACMG, the Evaluation of Genomic Applications in Practice and Prevention Working Group, the National Human Genome Research 
Institute, and others. These groups are providing guidance on which genomic results might be reportable and which clinical responses might be appropriate. ${ }^{64}$ Local committees with diverse expertise will need to adapt these guidelines to determine which results will be reported through institutional EHRs and what format these reports will take. Finally, the responsibility of providers may be shared with patients, who could be expected to access and review their own results through online patient portals.

\section{STORAGE AND REINTERPRETATION OF GENOMIC DATA}

Where and how to store the significant amount of data generated by genome sequencing is currently unresolved. One solution is to place only variants of established clinical utility in the EHR with linkage to clinical decision support. The entire genome sequence could be placed in a repository outside the EHR, with reinterrogation as needed. ${ }^{35}$ To enable such an approach, methods that allow EHR systems to interact with stored sequence data will be needed. It is also possible that with decreasing costs and improved reliability of genome sequencing, it may be cheaper and more reliable to simply resequence the genome if additional analyses are needed.

Another issue is the need for reinterpretation of variants of uncertain significance as new knowledge merges ${ }^{65}$ This could be a major concern with liability implications for the laboratory that generated the results, as well as the physician who ordered the test. For example, some investigators have suggested that physicians providing genomic consultation or counseling also have a duty to provide postgenomic test interpretation of results and a duty to prevent foreseeable harm. ${ }^{66,67}$ Posttest genetic counseling may also be considered part of this duty, although the exact role that counseling plays in a duty to reinterpret remains uncertain. Enforcing a duty to reinterpret future data or a duty to follow up with commercial genetic-testing companies may also pose its own set of challenges.

Although current policy in the United States does not explicitly require health professionals to initiate follow-up contact with patients after genetic tests, such an expectation could potentially be created through emerging standards of care or case law. ${ }^{68}$ Given that the integration of genomics into clinical care may depend on attaining clarity around this issue, a prospective approach to clarifying the provider's duty to rescue may be called for. Reasonable standards could be set through a combination of new regulations and/or new legislation. Guidance is needed, especially in the area of genomic research studies, where vast repositories of genetic information can be susceptible to evolving interpretation and reinterpretation.

A potential "secondary" use of EHR data is clinical research. How genomic data are stored will affect how they are accessed for clinical research. The majority of the public supports researcher access to and use of their genomic data for research purposes, provided employers and insurers put in place measures to prevent discrimination or exclusion from coverage based on preexisting conditions. ${ }^{69}$ Another point of debate is whether pharmaceutical companies that partner with insurance agencies should have access to genomic and phenotypic data that reside in EHRs. There is a need to develop a framework for the secondary use of EHR genomic and phenotypic data, including policies, standards, and best practices. ${ }^{69}$

\section{PRIVACY AND NONDOCUMENTATION}

Patient privacy is an important consideration in the adoption of EHRs. Although controversial, the practice of opting not to document sensitive information in a medical record is arguably as old as medical records themselves. There is some evidence, in particular, that nondocumentation of genomic information is more prevalent among patients with life-threatening conditions including Huntington disease, cancer, and other hereditary conditions. ${ }^{70,71}$ Given that neither the Health Insurance Portability and Accountability Act nor the Genetic Information Nondiscrimination Act have eliminated the risk of genetic discrimination ${ }^{72}$-nor the fear of $i^{73}$ - the interest in this practice is understandable. ${ }^{74}$ Patients and providers using EHRs may prefer a nondocumentation approach to certain genetic test results under certain circumstances.

Given that any clinical use of genomic data is likely to depend on electronic analysis, interpretation, and reporting of test results, results reported to patients and providers will be logged permanently. In place of nondocumentation, one could consider policies on the privacy features of EHRs to allow patients and their providers to set specific limitations on access to results they prefer to keep private. For example, patients may wish to limit access to their genomic status to specific classes of EHR users, or limit such results from transfer to EHR systems that interoperate with the originating institution. Medical record segmentation is one approach to provide privacy and protect against discrimination for certain medical conditions such as sexually transmitted disease, substance abuse, and mental health, by sequestering or redacting certain medical codes from a patient's record. ${ }^{75}$ Segmentation of sensitive genetic information has been recommended but raises important questions. ${ }^{72}$ For instance, which categories of sensitive information should be subject to segmentation? What, if any, electronic messages should alert clinicians that information has been segmented? Should clinical decision support rules be permitted to scan segmented information, and how does one prevent the decision support output from revealing segmented information? Should segmented information be made accessible in an emergency? What information should be disclosed to a third party pursuant to an authorization? ?2,74,76 $^{2}$

\section{DUTY TO WARN}

Integration of genomic information into EHRs complicates the issue of duty to warn, given the potential linkage to other relatives and the numerous physicians who are likely to have access to information without clarity on whose obligation it is to warn. There is a need for clearer interpretations of the duty to warn patients and their relatives, particularly in instances 
in which the physician has information that can forecast medical risks. The overriding responsibility of the healthcare provider is to the patient. Warning relatives over the objection of the patient is ethically dubious and in most cases violates the Health Insurance Portability and Accountability Act privacy rule. ${ }^{77}$ Furthermore, imposing the burden of the duty to warn on a physician alone is unrealistic. This is particularly true when one considers the number of relatives needed to contact and the amount of genetic counseling and education each relative would likely require to understand the results of genetic tests. Many have argued that clinicians have an ethical responsibility to at least "encourage" patients, in a noncoercive manner, to share genomic information with their families. ${ }^{67}$

\section{CONCLUSIONS}

It is important to work toward equitable access to genomic testing and EHRs with genomic clinical decision support to help reduce health-care disparities. The incorporation of genomic information into the EHR, although still in its infancy, raises important questions about patient autonomy, confidentiality, privacy, and the obligations of the physician. Implementation of genomics in the clinical setting will have to strike a fair balance between the mandate to improve health care and the need for reducing potential harms. Important questions that need to be addressed by professional organizations, clinicians, payers, and patients include what genomic results can be placed in the EHR, in what format they should be reported, and what expectation there will be for providers to follow up on new results. In addition, a distinction has to be drawn between deciding not to include in the EHR results that are delivered and not delivering them in the first instance. Obligations of confidentiality are owed only by health-care providers and health-care systems, but the patient should arguably be able to choose what information to disclose. Informatics and decision support are going to be critical to enable primary-care providers to use genomic medicine at point of care. There is a need to address the complexity of both delivering and protecting health information of all types in an electronic, interoperable world; this is an important prerequisite for a new era of genome-informed medicine.

\section{ACKNOWLEDGMENTS}

This work was supported by funding support from U01 HG006385, U01HG006378, and R01HG006844 to B.A.M. and E.W.C.; UL1 TR000135 from the National Center for Advancing Translational Sciences and R01CA154517 from the National Cancer Institute and the National Human Genome Research Institute (NHGRI) to B.A.K.; U01HG006382-01 from the Geisinger E-Genomic Medicine Program, NHGRI, Geisinger Health System, to M.S.W.; U01 HG006378, R01HG006844, and 1R21HG00612 to E.W.C.; U01HG006380 and the Charles Bronfman Institute for Personalized Medicine to S.C.S; and U01HG006379 to I.J.K.

\section{DISCLOSURE}

The authors declare no conflict of interest.

\section{REFERENCES}

1. Green ED, Guyer MS; National Human Genome Research Institute. Charting a course for genomic medicine from base pairs to bedside. Nature 2011;470:204213.

2. McGuire AL, Cho MK, McGuire SE, Caulfield T. Medicine. The future of personal genomics. Science 2007;317:1687

3. Hoffman MA, Williams MS. Electronic medical records and personalized medicine. Hum Genet 2011;130:33-39.

4. Worthey EA, Mayer AN, Syverson GD, et al. Making a definitive diagnosis: successful clinical application of whole exome sequencing in a child with intractable inflammatory bowel disease. Genet Med 2011;13:255-262.

5. Mardis ER, Wilson RK. Cancer genome sequencing: a review. Hum Mol Genet 2009;18(R2):R163-R168.

6. Mardis ER. Cancer genomics identifies determinants of tumor biology. Genome Biol 2010;11:211.

7. Cheng L, Lu W, Kulkarni B, et al. Analysis of chemotherapy response programs in ovarian cancers by the next-generation sequencing technologies. Gynecol Oncol 2010;117:159-169.

8. Kreso A, O'Brien CA, van Galen P, et al. Variable clonal repopulation dynamics influence chemotherapy response in colorectal cancer. Science 2013;339:543548.

9. Link DC, Schuettpelz LG, Shen D, et al. Identification of a novel TP53 cancer susceptibility mutation through whole-genome sequencing of a patient with therapy-related AML. JAMA 2011;305:1568-1576.

10. McDermott U, Downing JR, Stratton MR. Genomics and the continuum of cancer care. N Engl J Med 2011;364:340-350.

11. lyer $G$, Hanrahan AJ, Milowsky MI, et al. Genome sequencing identifies a basis for everolimus sensitivity. Science 2012;338:221.

12. Kullo IJ, Jarvik GP, Manolio TA, Williams MS, Roden DM. Leveraging the electronic health record to implement genomic medicine. Genet Med 2013;15:270-271.

13. Manolio TA, Chisholm RL, Ozenberger B, et al. Implementing genomic medicine in the clinic: the future is here. Genet Med 2013;15:258-267.

14. Evans JP. Health care in the age of genetic medicine. JAMA 2007;298:26702672.

15. Jha AK, DesRoches CM, Campbell EG, et al. Use of electronic health records in U.S. hospitals. N Engl J Med 2009;360:1628-1638.

16. Ash JS, McCormack JL, Sittig DF, Wright A, McMullen C, Bates DW. Standard practices for computerized clinical decision support in community hospitals: a national survey. J Am Med Inform Assoc 2012;19:980-987.

17. Marcotte L, Seidman J, Trudel K, et al. Achieving meaningful use of health information technology: a guide for physicians to the EHR incentive programs. Arch Intern Med 2012;172:731-736

18. de Ligt J, Willemsen $\mathrm{MH}$, van Bon BW, et al. Diagnostic exome sequencing in persons with severe intellectual disability. N Engl J Med 2012;367:1921-1929.

19. Berg JS, Adams M, Nassar N, et al. An informatics approach to analyzing the incidentalome. Genet Med 2013;15:36-44.

20. Sax U, Schmidt S. Integration of genomic data in electronic health recordsopportunities and dilemmas. Methods Inf Med 2005;44:546-550.

21. McClellan KA, Avard D, Simard J, Knoppers BM. Personalized medicine and access to health care: potential for inequitable access? Eur J Hum Genet 2013;21:143-147.

22. Collins F. Opportunities and challenges for the $\mathrm{NIH}$-an interview with Francis Collins. Interview by Robert Steinbrook. N Engl J Med 2009;361:1321-1323.

23. Personalized health care. 2008. http://www.hhs.gov/myhealthcare/. Accessed 18 December 2012.

24. Icahn School of Medicine at Mount Sinai. Whole Genome Sequencing and You. 2012. https://www.youtube.com/watch?v=IXamRS85hXU\&hd=1.

25. Delbanco T, Walker J, Bell SK, et al. Inviting patients to read their doctors' notes: a quasi-experimental study and a look ahead. Ann Intern Med 2012;157:461470.

26. About $A B G C$. http://www.abgc.net/ABGC/AmericanBoardof GeneticCounselors.asp. Accessed 2 April 2013.

27. Giarelli E, Reiff M. Genomic literacy and competent practice: call for research on genetics in nursing education. Nurs Clin North Am 2012;47:529-545.

28. Galbraith KL. What's so meaningful about meaningful use? Hastings Cent Rep 2013;43:15-17.

29. Juengst ET, Flatt MA, Settersten RA Jr. Personalized genomic medicine and the rhetoric of empowerment. Hastings Cent Rep 2012;42:34-40.

30. Brierley KL, Blouch E, Cogswell W, et al. Adverse events in cancer genetic testing medical, ethical, legal, and financial implications. Cancer J 2012;18:303-309. 
31. Thurston VC, Wales PS, Bell MA, Torbeck L, Brokaw JJ. The current status of medical genetics instruction in US and Canadian medical schools. Acad Med 2007:82:441-445.

32. Riley BD, Culver JO, Skrzynia C, et al. Essential elements of genetic cancer risk assessment, counseling, and testing: updated recommendations of the National Society of Genetic Counselors. J Genet Couns 2012;21:151-161.

33. Hofman KJ, Tambor ES, Chase GA, Geller G, Faden RR, Holtzman NA. Physicians' knowledge of genetics and genetic tests. Acad Med 1993;68:625632.

34. Fargher EA, Eddy C, Newman W, et al. Patients' and healthcare professionals' views on pharmacogenetic testing and its future delivery in the NHS Pharmacogenomics 2007;8:1511-1519.

35. Starren J, Williams MS, Bottinger EP. Crossing the omic chasm: a time for omic ancillary systems. JAMA 2013;309:1237-1238.

36. Westbrook MJ, Wright MF, Van Driest SL, et al. Mapping the incidentalome: estimating incidental findings generated through clinical pharmacogenomics testing. Genet Med 2013;15:325-331.

37. Ormond KE, Wheeler MT, Hudgins $L$, et al. Challenges in the clinical application of whole-genome sequencing. Lancet 2010;375:1749-1751.

38. Biesecker LG. Opportunities and challenges for the integration of massively parallel genomic sequencing into clinical practice: lessons from the ClinSeq project. Genet Med 2012;14:393-398.

39. Leventhal KG, Tuong W, Peshkin BN, et al. "Is it really worth it to get tested?": primary care patients' impressions of predictive SNP testing for colon cancer. J Genet Couns 2013;22:138-151.

40. Scheuner MT, Edelen MO, Hilborne LH, Lubin IM. Effective communication of molecular genetic test results to primary care providers. Genet Med 2013;15:444-449.

41. Scheuner MT, Hilborne L, Brown J, Lubin IM; members of the RAND Molecular Genetic Test Report Advisory Board. A report template for molecular genetic tests designed to improve communication between the clinician and laboratory. Genet Test Mol Biomarkers 2012;16:761-769.

42. Bitton A, Flier LA, Jha AK. Health information technology in the era of care delivery reform: to what end? JAMA 2012;307:2593-2594.

43. Health Information Exchange (HIE): Interoperability Basics Training Course. http://www.healthit.gov/providers-professionals/interoperability-basicstraining-courses. Accessed 11 March 2013.

44. Ruotsalainen P, Blobel B, Nykänen P, Seppälä A, Sorvari H. Framework model and principles for trusted information sharing in pervasive health. Stud Health Technol Inform 2011;169:497-501.

45. Boxwala AA, Kim J, Grillo JM, Ohno-Machado L. Using statistical and machine learning to help institutions detect suspicious access to electronic health records. J Am Med Inform Assoc 2011;18:498-505.

46. Rothstein MA. The Hippocratic bargain and health information technology. J Law Med Ethics 2010;38:7-13.

47. Green RC, Berg JS, Grody WW, et al. ACMG recommendations for reporting of incidental findings in clinical exome and genome sequencing. Genet Med 2013;15(10):294-300.

48. Gottesman O, Kuivaniemi H, Tromp G, et al. The Electronic Medical Records and Genomics (eMERGE) Network: past, present, and future. Genet Med 2013; .

49. Scott CT, Caulfield T, Borgelt E, llles J. Personal medicine-the new banking crisis. Nat Biotechnol 2012;30:141-147.

50. Pulley JM, Denny JC, Peterson JF, et al. Operational implementation of prospective genotyping for personalized medicine: the design of the Vanderbilt PREDICT project. Clin Pharmacol Ther 2012;92:87-95.

51. Hicks JK, Crews KR, Hoffman JM, et al. A clinician-driven automated system for integration of pharmacogenetic interpretations into an electronic medical record. Clin Pharmacol Ther 2012;92:563-566.

52. O'Donnell PH, Bush A, Spitz J, et al. The 1200 patients project: creating a new medical model system for clinical implementation of pharmacogenomics. Clin Pharmacol Ther 2012;92:446-449.

53. Chen ES, Melton GB, Engelstad ME, Sarkar IN. Standardizing Clinical Document Names Using the HL7/LOINC Document Ontology and LOINC Codes. AMIA Annu Symp Proc 2010;2010:101-105.
54. Facio FM, Sapp JC, Linn A, Biesecker LG. Approaches to informed consent for hypothesis-testing and hypothesis-generating clinical genomics research. $B M C$ Med Genomics 2012;5:45

55. Murphy J, Scott J, Kaufman D, Geller G, LeRoy L, Hudson K. Public expectations for return of results from large-cohort genetic research. Am J Bioeth 2008;8:3643.

56. Facio FM, Brooks S, Loewenstein J, Green S, Biesecker LG, Biesecker BB. Motivators for participation in a whole-genome sequencing study: implications for translational genomics research. Eur J Hum Genet 2011;19:1213-1217.

57. Allyse M, Michie M. Not-so-incidental findings: the ACMG recommendations on the reporting of incidental findings in clinical whole genome and whole exome sequencing. Trends Biotechno/ 2013;31:439-441.

58. Cho MK. Understanding incidental findings in the context of genetics and genomics. J Law Med Ethics 2008;36(2):280-285, 212.

59. Clayton EW, McGuire AL. The legal risks of returning results of genomics research. Genet Med 2012;14:473-477.

60. Richardson HS, Cho MK. Secondary researchers' duties to return incidental findings and individual research results: a partial-entrustment account. Genet Med 2012:14:467-472.

61. Wolf SM. The past, present, and future of the debate over return of research results and incidental findings. Genet Med 2012;14:355-357.

62. Morreim EH. The clinical investigator as fiduciary: discarding a misguided idea. $J$ Law Med Ethics 2005;33:586-598.

63. Ossorio P. Taking aims seriously: repository research and limits on the duty to return individual research findings. Genet Med 2012;14:461-466.

64. Kohane IS, Masys DR, Altman RB. The incidentalome: a threat to genomic medicine. JAMA 2006:296:212-215.

65. Pyeritz RE. The coming explosion in genetic testing-is there a duty to recontact? N Engl J Med 2011;365:1367-1369.

66. Cancer Genetics Risk Assessment and Counseling (PDQ): Ethical, Legal, and Social Implications. http://www.cancer.gov/cancertopics/pdq/genetics/riskassessment-and-counseling/HealthProfessional/page6. Accessed 28 March 2013.

67. Offit K, Groeger E, Turner S, Wadsworth EA, Weiser MA. The "duty to warn" a patient's family members about hereditary disease risks. JAMA 2004;292:14691473

68. Rothstein MA, Siegal G. Health information technology and physicians duty to notify patients of new medical developments. Hous J Health L Policy 2012;12(2):93-136

69. Safran C, Bloomrosen M, Hammond WE, et al. Toward a national framework for the secondary use of health data: an American Medical Informatics Association White Paper. J Am Med Inform Assoc 2007;14:1-9.

70. Bunnik EM, Schermer MH, Janssens AC. Personal genome testing: test characteristics to clarify the discourse on ethical, legal and societal issues. BMC Med Ethics 2011:12:11.

71. Diergaarde B, Bowen DJ, Ludman EJ, Culver JO, Press N, Burke W. Genetic information: special or not? Responses from focus groups with members of a health maintenance organization. Am J Med Genet A 2007;143:564-569.

72. Rothstein MA. Putting the Genetic Information Nondiscrimination Act in context. Genet Med 2008;10:655-656.

73. Allain DC, Friedman S, Senter L. Consumer awareness and attitudes about insurance discrimination post enactment of the Genetic Information Nondiscrimination Act. Fam Cancer 2012;11:637-644.

74. Klitzman R. Exclusion of genetic information from the medical record: ethical and medical dilemmas. JAMA 2010;304:1120-1121.

75. Chan EM, Lam PE, Mitchell JC. Understanding the challenges with medical data segmentation for privacy. Health Tech'13, 2013. https://www.usenix. org/conference/healthtech13/understanding-challenges-medical-datasegmentation-privacy.

76. Rothstein MA. Currents in contemporary bioethics. Access to sensitive information in segmented electronic health records. J Law Med Ethics 2012;40:394-400.

77. Professional disclosures of familial genetic information. The American Society of Human Genetics Social Issues Subcommittee on Familial Disclosure. Am J Hum Genet 1998:62(2):474-483. 